\title{
Evolución y uso del agua de riego en los sistemas campesinos de producción de leche del noroeste del Estado de México
}

\section{Evolution and usage of irrigation water in the farmer milk production systems from North-East Estado de Mexico}

\author{
Tirzo Castañeda-Martínez \\ SERgio Franco-MaAss \\ Carlos GonzÁlez-Esquivel \\ Angélica Espinoza-Ortega*
}

\begin{abstract}
In this paper we analyse retrospectively the evolution of irrigation systems and the agricultural activity in the North-East region of Estado de Mexico. We have employed several analytical strategies starting from the information provided by governmental departments, interviews with key informers as well as surveys of the producers that used this resource. We identified that the availability of water has contributed to the establishment of foraging crops mainly destined to the feeding of milking cows, given that this is the livestock platform of the region. The production of milk together with the cultivation of basic crops such as maize have contributed to the integration of the agriculture and livestock industries.
\end{abstract}

Keywords: unit of agricultural production, irrigation, agriculture and livestock activities, maize production, milk production.

\section{Resumen}

Se analiza en retrospectiva, la evolución del sistema de riego y la actividad agropecuaria en la región noroeste del estado de México. Se emplearon diversas estrategias de análisis a partir de información proporcionada por dependencias gubernamentales, entrevistas a informantes clave, así como la aplicación de cuestionarios a productores que hicieron uso del recurso. Se logró identificar que la disponibilidad de agua ha contribuido al establecimiento de cultivos forrajeros orientados principalmente a la alimentación de vacas lecheras, dado que constituyen el eje pecuario de la región.

Palabras clave: unidad de producción agrícola, riego, actividad agropecuaria, producción de maíz, producción de leche.

*Universidad Autónoma del Estado de México. Correos-e: tirzo24@yahoo.com.mx, Serfm@uamex.mx,cge1@uamex.mx, aeo@uamex.mx. 


\section{Introducción}

La agricultura en México ha transitado por diversas etapas a través del tiempo. Una de las más relevantes se presentó con la crisis internacional de 1929-1932, posteriormente entre 1940 y 1958 se identificó un periodo de auge agrícola como consecuencia de las reformas que realizó el gobierno cardenista, el cual se explicó por tres factores fundamentales (Gómez, 1996: 37-40): 1) la reforma agraria que desintegró el monopolio latifundista permitió la inversión en agricultura, aportó la base social y la organización de recursos productivos como la tierra y el agua, 2) la inversión pública en obras de irrigación, incorporando los recursos naturales a la producción, incrementó la productividad de las tierras agrícolas y favoreció el desarrollo tecnológico y 3) el comportamiento favorable de los precios agrícolas que generaron la capitalización de la agricultura.

A partir de 1958 se acentuó el énfasis en la industrialización como motor de desarrollo económico y el proceso de sustitución de importaciones mediante el proteccionismo comercial, con aranceles elevados y cuotas de importación en numerosos productos. La justificación del modelo industrial se basó en que éste generaría un efecto de arrastre capaz de estimular el crecimiento de la agricultura y los demás sectores económicos, mientras que los efectos negativos se podrían compensar a través de apoyos a la producción; es decir, si la agricultura no se podía alentar vía mayores precios, sí a través de menores costos (Villalobos, 2000). En el proceso se estimuló la actividad agrícola a través de programas de inversión pública, asistencia técnica, así como mecanismos para reducir el costo del crédito, insumos y riego.

En la agricultura empresarial la aplicación de esta política generó el desarrollo de un patrón tecnológico que ha utilizado recursos costosos para el país, como el uso de maquinaria agrícola y fertilizantes. Respecto al riego se generalizaron técnicas que han empleado elevados coeficientes de agua, pero con bajos costos en infraestructura propia y de operación, ya que el costo real del agua no se reflejó en los costos del agricultor, lo que originó graves deficiencias en el mantenimiento de la infraestructura de riego (Gómez, 1996; Vargas, 2002).

En el caso de los pequeños productores, de maíz y frijol principalmente, han requerido de algún ingreso extra para costear los desembolsos del proceso de producción y consumo, por lo que la alternativa más accesible es el trabajo asalariado fuera de 
la explotación familiar, donde es de suma importancia el salario generado fuera de la unidad de producción y el precio del maíz producido (Barkin, 2003).

A partir de 1982 la producción agrícola se orientó hacia un modo de producción sustentado en políticas neoliberales y con el gobierno de Carlos Salinas de Gortari se llevó a cabo un programa de modernización para todo el sector productivo del país. Respecto de los recursos hidráulicos, se implementó la transferencia de los distritos de riego a las asociaciones civiles de usuarios, con el objeto de mejorar la productividad de los recursos agua y suelo, así como alcanzar una agricultura sostenible con la participación de los usuarios y los gobiernos estatales (Dávila et al., 1996).

Las respuestas sociales han sido diferentes, por un lado se encuentra la agricultura empresarial inserta en las grandes tendencias mundiales de cambio productivo y, por otro, un amplio sector de productores que han modificado sus estrategias de subsistencia para lograr sobrevivir (Vargas, 2002), lo que plantea enormes retos en cuanto a los rezagos en la gestión del agua de riego, así como en el ordenamiento de usos y derechos.

Los datos más recientes mostraron que en México la agricultura se practicó en una superficie aproximada de 20 millones de hectáreas, de las cuales 6.3 millones utilizaron sistemas de irrigación (FAO, 2004). De la superficie irrigada, 3.3 millones de hectáreas correspondieron a 80 distritos de riego y 2.9 millones a 30 mil unidades de mediano y pequeño riego para el desarrollo rural. La tierra irrigada representó 30\% de la superficie total del país destinada a la agricultura, generó cerca de $50 \%$ del valor de la producción agrícola total y más de $30 \%$ de los empleos del sector (Villagómez, 2002).

Aunado a lo anterior, la producción de alimentos, tal como lo considera la Organización de las Naciones Unidas para la Agricultura y la Alimentación (FAO), depende de la disponibilidad de recursos hídricos apropiados y sostenibles, dado que el agua de riego aumenta los rendimientos de todos los cultivos entre $100 \mathrm{y}$ 400\%. La agricultura utiliza 70\% del total de agua disponible y en muchos países en desarrollo la cifra es de 85 a 95\%, con el inconveniente de que el agua de riego se extrae más rápido de lo que se repone (Zetina, 2002).

Dado el carácter multidimensional que presenta la agricultura, se deben considerar los distintos factores que intervienen en ella, así como las características propias de cada territorio. De 
acuerdo con Cordero et al. (2003), en un territorio confluyen e interactúan factores provenientes de cuestiones macroeconómicas, espaciales y microeconómicas que permiten la competitividad, su producción, así como la posibilidad de promover la cohesión al interior del mismo y con el resto de la economía nacional. La economía rural depende y se estructura de acuerdo con los recursos disponibles en el territorio, por lo que actividades relacionadas directamente con recursos naturales van a definir el modelo productivo. En este sentido se pueden determinar cuatro niveles.

1) El nivel meta determinado por la capacidad de conducción nacional comprende elementos socioculturales, valores, así como organización política, económica y jurídica.

2) El nivel macro en el que intervienen la estabilidad y seguridad de las condiciones macroeconómicas que afectan al espacio territorial, así como la capacidad del país de relacionarse internacionalmente.

3) El nivel meso donde sobresalen factores como las distancias, la infraestructura y la base de recursos naturales.

4) El nivel micro que se refiere a aspectos relacionados con el ámbito empresarial o la unidad de producción, considerando elementos como precio, calidad y factores espaciales que resultan condicionantes.

En la región noroeste del Estado de México, a nivel meso destacó el agua de riego y su papel fundamental en los sistemas de producción agropecuarios locales. Lo anterior a través de la creación de infraestructura e implementación de cultivos forrajeros dirigidos a la alimentación de vacas lecheras que constituyen la principal actividad pecuaria de la zona. El tipo de ganado predominante es la raza holstein, cruzas de suizo-holstein en ocasiones y el tipo criollo en menor cantidad. Los factores mencionados tuvieron su efecto a nivel micro en la unidad de producción donde se llevó a cabo la utilización del agua de acuerdo con las necesidades de cada explotación agropecuaria. La región presentó la mayor superficie irrigada en el Estado de México, sin embargo, no hay estudios que analicen con detalle cómo ha sido su evolución y su situación actual, por lo que el trabajo tuvo como primer objetivo analizar la evolución del riego y la actividad agropecuaria en la zona, mediante un análisis retrospectivo regional de 1930 a 2000. 


\section{Figura I}

\section{Localización de la región noroeste}

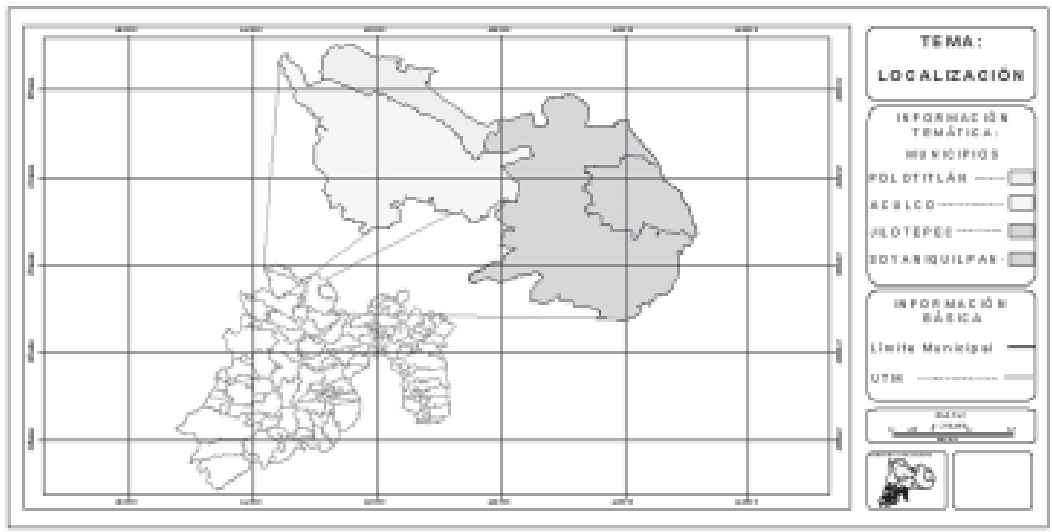

Fuente: Elaboración propia con información del INEGI, 2000.

El segundo objetivo consistió en determinar los usos del agua de riego en las unidades de producción, así como la actividad agrícola y pecuaria que se desarrolló en función de la utilización del mismo.

\section{Metodología}

El trabajo se llevó a cabo en cuatro de los siete municipios que comprende la región noroeste del Estado de México: Aculco, Polotitlán, Soyaniquilpan de Juárez y Jilotepec.

En el estudio se evaluaron sólo dos niveles de análisis: a) regional, que comprendió cuestiones relacionadas con el nivel meso considerando factores espaciales como el agua de riego e infraestructura en función de las distintas presas y canales de distribución del agua, y b) nivel microeconómico, que consideró la unidad de producción para analizar cuál fue la utilización del recurso.

La evolución del riego y la actividad agropecuaria que se generó a través del tiempo se analizó con información de dependencias gubernamentales como el INEGI a partir de los censos agrícolas-ganaderos de 1937, 1956, 1965, 1970 y 1994, así como la carta temática del 2000, la Sedagro, la CNA y entrevistas con informantes clave.

Para evaluar los usos del agua de riego en las unidades de producción se tomó como marco de muestra los 5,236 productores registrados ante la CNA con derecho a riego en la zona de estudio. El tamaño de muestra fue de $5 \%$, de acuerdo con lo que 
sugieren Segura y Honhold (1993), por lo que el número de productores entrevistados fue de 262.

Dado que la CNA no facilitó la base de datos de los usuarios del riego por cuestiones de seguridad, se optó por un muestreo por conveniencia, ya que un aleatorio no fue posible, además de que el estudio basó el instrumento de medida en el riego y no en los individuos, por lo que las posibles inferencias a la población son válidas (Daniel, 2004).

Para obtener la información a través de las encuestas se procedió a realizar un recorrido en cada comunidad, se invitó a los productores a participar en el estudio y la única condición fue que contaran con derecho a riego en sus parcelas en cualquiera de sus formas, es decir, como usuario directo, por sucesión de los padres, renta de terrenos con riego, compra o eventuales. El promedio de cuestionarios aplicados fue de ocho por comunidad y no se regresó nuevamente al mismo lugar para evitar sesgos en la información.

El número de usuarios de riego registrados ante la CNA también fue diferente para cada municipio que se muestreó, por lo que se decidió realizar una proporción de productores que hicieron uso en cada municipio. En Jilotepec se entrevistó a 86 productores, 24 en Soyaniquilpan de Juárez, 107 en Aculco y 45 en Polotitlán. La información se analizó con estadística descriptiva.

El cuestionario consideró tres apartados principales: 1) información que evaluó la superficie agrícola total, superficie sembrada, tenencia de la tierra, renta, tipo de cultivos y destino de los mismos, 2) información sobre el riego que consideró la superficie irrigada, tipo de riego para los cultivos, usos del riego, cantidad de agua destinada para cada cultivo, costos, manejo del agua y su relación con las asociaciones de usuarios, 3) información pecuaria donde se registró el tipo de animales en la unidad de producción, cultivos destinados por especie, su importancia en la explotación familiar y la producción de leche en caso de que la unidad de producción contara con vacas.

\section{Resultados y discusión}

\subsection{Evolución de la agricultura de riego}

En la región noroeste del Estado de México, al igual que en el resto del país, las haciendas concentraron los recursos para la producción agropecuaria: agua, bosques y pastizales (Salinas, 
2001). En la zona de estudio destacaron tres haciendas, Arroyo Zarco que se ubicó en el municipio de Aculco y llegó a contar con 47,000 ha, José el Marqués en Jilotepec con 14,191 ha (Aguado, 1998) y la Goleta en Soyaniquilpan de Juárez con una extensión de 10,498 ha. Esta última formó parte de un latifundio conformado por otras haciendas (San Antonio Calpulalpan y Tandejé), con una superficie de 43,654 ha en conjunto (Morales, 1998; Salinas, 2001).

Las haciendas se dedicaron a la producción de maíz, trigo, frijol, haba y arvejón como cultivos básicos, mientras que la actividad ganadera se caracterizó por la engorda de reses en pastos nativos en épocas de lluvia, además de otros animales como borregos, bueyes para las labores agrícolas y vacas criollas. A partir de 1921 las haciendas fueron fraccionadas con el reparto agrario y permitió a una mayor población el acceso a tierra para el establecimiento de labores agrícolas.

Respecto al riego, hasta principios del siglo xx la construcción de cuerpos de agua en el país se llevó a cabo por la iniciativa privada y fue hasta 1926 que se impulsó en México una verdadera política de riego, creándose la Comisión Nacional de Irrigación (CNI) con el objetivo de incrementar las áreas irrigadas (Arredondo, 1971), así como resolver el abastecimiento de materias primas y alimentos a través de la construcción de obras hidráulicas financiadas por el gobierno federal. La CNI emprendió la construcción de presas y canales de distribución de agua, con lo que estableció en México los primeros distritos de riego (Anaya, 1975). A la par de los distritos de riego, en 1936 se llevó a cabo la construcción de pequeñas obras de irrigación con áreas de extensión moderada para cultivos que se entregaron a los usuarios para su operación directa, a las cuales se les dio el nombre de pequeña irrigación (Gómez, 1994).

Como parte de esta política, en la región noroeste del Estado de México se creó infraestructura y en 1938 entró en operación la presa Huapango, una de las más importantes del estado que forma parte de la cuenca Río Moctezuma-Pánuco y se ubica entre los municipios de Aculco y Jilotepec. Años después, en 1951 comenzó a funcionar Danxho, presa que irriga a los municipios de Jilotepec y Soyaniquilpan de Juárez. También existen otras presas de menor tamaño construidas a través del tiempo y distribuidas al interior de la región (Molino, Nado y la Goleta) con la misma finalidad de abastecer de agua para la actividad agropecuaria. 
En relación con el clima, el que predomina es el templado subhúmedo con lluvias en verano de mayor humedad (C(w2) (INEGI, 1997), cuya precipitación pluvial presenta un rango de 900 $\mathrm{mm}$ en la parte sur de montañas a $600 \mathrm{~mm}$ anuales en la zonas más secas, por lo que la creación de presas es fundamental para irrigar las partes más áridas como Polotitlán, norte de Jilotepec y Soyaniquilpan.

De acuerdo con los censos agropecuarios, la superficie irrigada mostró un promedio de 13,826.2 ha de 1930 a 1990, sin embargo, en la década de los treinta se cubrió 15.4\% del total irrigado entre el periodo mencionado, para los cuarenta 22.5\%, 26\% en 1960 y comenzó su descenso en 1970 con 23.6\%, de forma que para 1990 el porcentaje de irrigación disminuyó a sólo 12.5\%. Lo anterior mostró la incongruencia de los datos de los censos, dado que se presentó una menor superficie irrigada cuando se contó con presas, en relación con el tiempo en que aún no iniciaban operación las mismas. El mayor porcentaje de irrigación durante la década de los sesenta se relacionó con la mayor precipitación pluvial de 1958 a 1960 y, por tanto, la captación de agua en las presas de la zona.

En 1990 se llevó a cabo la transferencia de los distritos de riego en el país y por ende en la zona. De acuerdo con lo que informó la CNA se irrigó una superficie promedio de 10,419.8 ha entre 1991 y 2003, sin embargo, se consideró sólo la superficie irrigada con las presas que conforman los dos distritos de riego de la zona y faltó agregar lo que se irriga con las presas catalogadas como unidades de riego que se distribuyen al interior de la región. No obstante, en este trabajo no fue posible incorporar la información por la falta de estadísticas en las distintas dependencias involucradas en la gestión del agua de riego, aunado al periodo que duró la reestructuración en el manejo del agua que pudo afectar la recopilación de datos reales por parte de la CNA y las mesas directivas de las asociaciones de usuarios.

El objetivo inicial del aprovechamiento del agua de riego fue para producir cultivos básicos, de los cuales el maíz ha sido el más importante: en 1930 se destinaron 13,027 ha a su producción y en 1990, 26,377.7 ha, con un incremento de 49.4\%. Tradicionalmente el cultivo se utilizó para autoconsumo, no obstante, al pasar el tiempo y dado el bajo precio del maíz, los productores optaron por destinarlo a la alimentación de los animales. Otro cultivo importante fue el trigo, en 1930 se cultivaron 2,426 ha, sin embargo, para 1990 presentó un decremento 


\section{Gráfica I}

Superficie de maíz y forrajes de 1930 a 1990

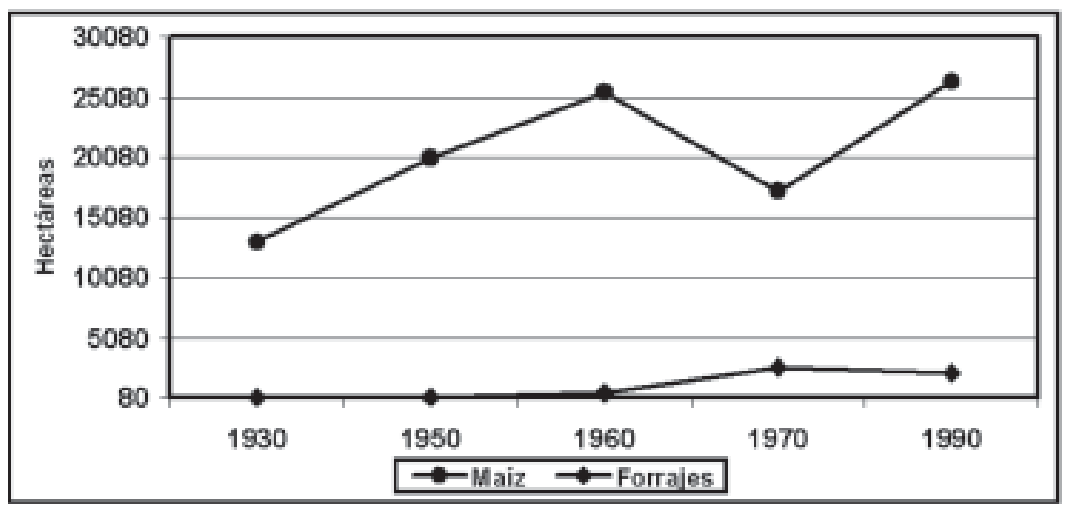

Fuente: Elaboración propia con información de los censos agrícolas-ganaderos de 1930, 1950, 1960, 1970 y 1990.

de 70\%, ya que sólo se reportaron 723.3 ha. El frijol y el haba complementaron el resto de cultivos básicos, aunque se han intercalado con maíz o se han sembrado en muy poca proporción.

El cambio en el patrón de cultivos se empezó a apreciar en la década de los sesenta con el gobierno de Juan Fernández Albarrán (Espinoza, 2004), a partir de los programas de impulso a la producción de forrajes mediante praderas inducidas de gramíneas y leguminosas. Esta situación se reflejó en el censo de 1970 que reportó 1,003 ha de praderas establecidas en la región. Otro forraje que destacó fue la avena, aunque su crecimiento fue posterior, en 1970 se destinaron únicamente 55 ha y para 1990 se reportaron 2,161. Este rápido crecimiento fue posible por el ciclo corto del cultivo, sembrado en los terrenos cosechados de maíz y cultivado regularmente en el periodo seco, en los meses de octubre-noviembre, lo que permite aprovechar mejor el terreno agrícola y es precisamente la existencia del riego lo que hace factible su producción. Respecto a la alfalfa, su momento de auge fue entre 1960 y 1970.

Al analizar los cambios entre la producción de maíz y la de forrajes se observó que existe una relación inversamente proporcional, lo que indica que los forrajes son una respuesta a la problemática del maíz y a la existencia de animales que aprovechan su producción.

La producción de forrajes necesariamente está ligada al sector pecuario y en ese sentido destacó la producción de bovinos lecheros. De acuerdo con Espinoza (2004), en los años cincuen- 
ta se impulsó la producción de leche en la región mediante la creación de una cuenca lechera y la organización de productores. En 1960 el gobierno estatal promovió la producción de forrajes, así como la red de canales de distribución del agua de riego, además de acciones locales como la creación de la Unión de Productores de Leche de Polotitlán en 1962, que en conjunto estimularon la actividad lechera de la zona.

Lo anterior se reflejó al analizar los datos de los censos agropecuarios, dado que el número de cabezas de ganado bovino se incrementó $71.7 \%$ de 1930 a 1990, registrando el despunte de su crecimiento a partir de 1960, década en que se intensificó la producción de leche en la zona.

El municipio de Jilotepec concentró el mayor número de bovinos a través del tiempo, en 1930 contó con $60.1 \%$ del total de la región, 53.6\% en 1960 y 42.8\% en 1990. Aculco fue el segundo municipio en importancia en este rubro con $23 \%, 18 \%$ y $31.2 \%$, respectivamente. Le siguieron en orden de importancia Polotitlán y Soyaniquilpan de Juárez.

Desafortunadamente, no contar con un censo agropecuario más reciente dificultó el análisis hasta el periodo actual, por lo que se tuvo que recurrir a la única información disponible. De acuerdo con la Sedagro (2000a), el número de bovinos mostró una disminución importante para 1998, ya que se reportaron 27,618 cabezas, lo que representó un decremento de $57.5 \%$ en comparación con el total de 1990 (47,992 bovinos). No obstante esta situación, para las queserías de la zona no hay un decremento en el abasto de leche, por lo que se puede generar la hipótesis de que es posible que los rendimientos de leche por animal se hayan incrementado, ya que también han existido programas gubernamentales dirigidos a mejorar la calidad genética de los animales por medio de la introducción de ganado mejorado e inseminación artificial.

Actualmente la región cuenta con 213,327 ha de superficie de labor (Sedagro, 2000b), de las cuales 38\% se destinó a actividades agrícolas, $26 \%$ a pecuarias, $28 \%$ a la explotación forestal y $8 \%$ a otros usos. Considerando sólo superficie agrícola $(80,290$ ha), $45 \%$ fue irrigada a través de presas y 55\% correspondió a la superficie de temporal.

De las diferentes presas se desprenden varios canales de distribución del agua denominados principales y laterales. La construcción de canales de riego se ha realizado por medio de la Sedagro que otorga el financiamiento y los productores que aportan 
Figura II

Cabezas de ganado bovino por municipio

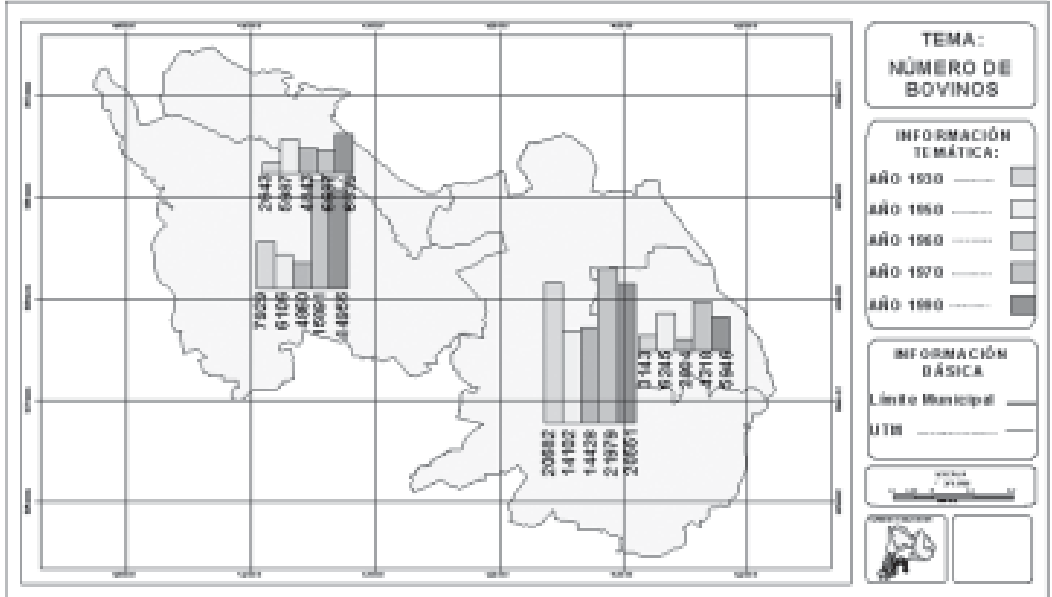

Fuente: Elaboración propia con datos de los censos agrícolas-pecuarios de 1930, 1950, 1960,1970 y 1990.

Figura III

Uso del suelo en la región noroeste

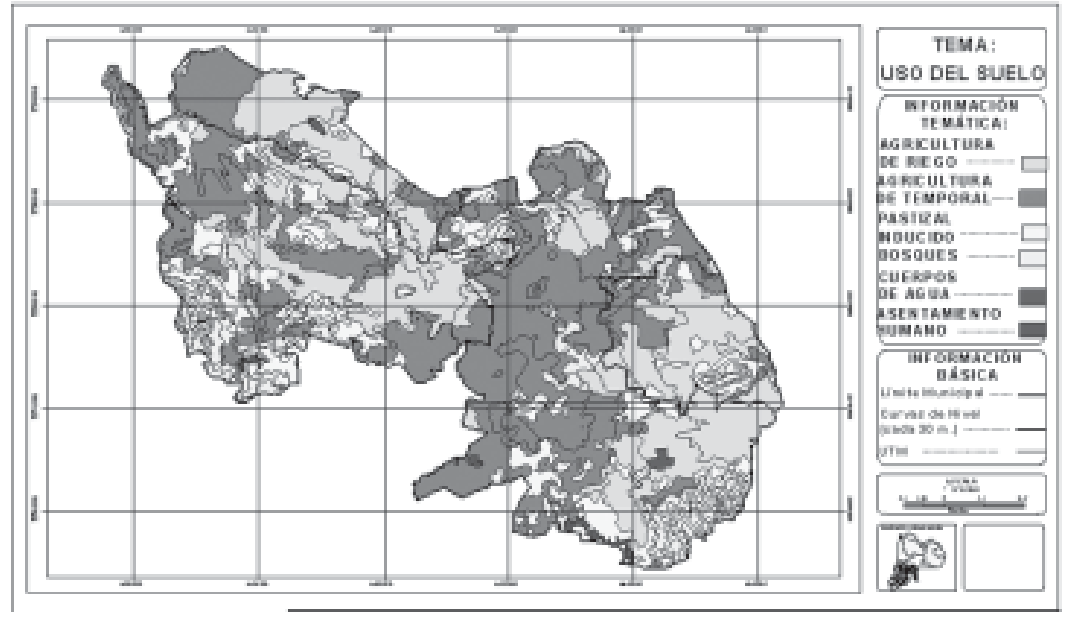

Fuente: Elaboración propia con información del INEGI, 2000. 
Figura IV

Principales canales de distribución del agua de riego

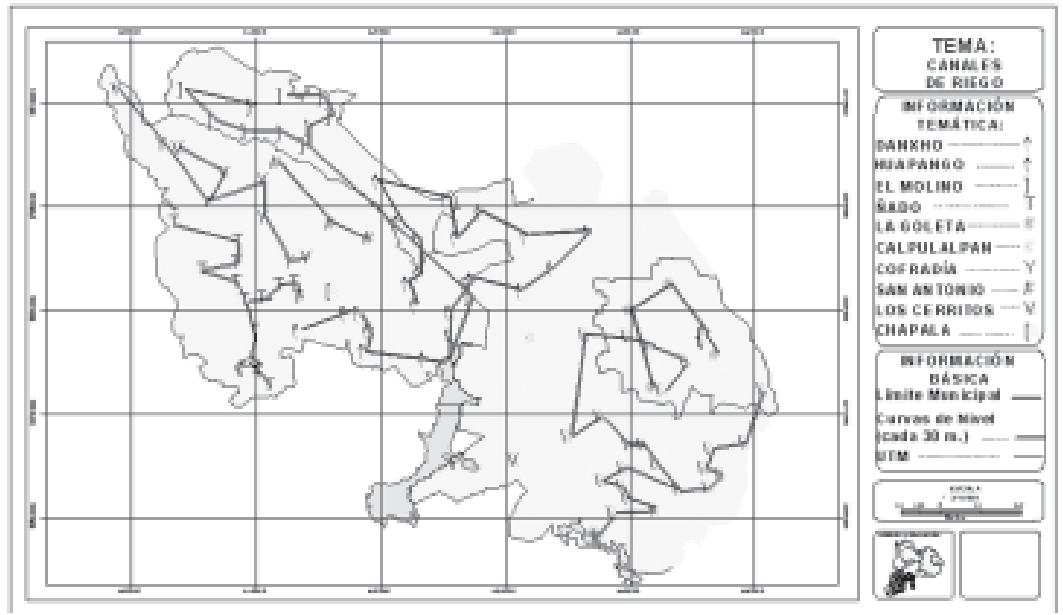

Fuente: Elaboración propia con información del INEGI, 2000 y la CNA, 2003.

la mano de obra, con la finalidad de evitar el desperdicio de agua por filtración, disminuir las labores de desasolve y el tiempo que tarda el agua en llegar de las presas a las unidades de producción, el cual se reduce en horas e incluso días.

\subsection{Usos del agua de riego en las unidades de producción}

Si bien los objetivos de este artículo no consideraron un análisis particular de la transferencia de los distritos de riego, actualmente es el mecanismo con que cuentan los productores para obtener el recurso agua en sus parcelas, por lo que se describe brevemente la situación actual en la región en relación con el manejo del riego y la actividad agrícola que prevalece.

Por política nacional, a partir de 1990 se llevó a cabo la transferencia de los distritos de riego en el país y se formaron las asociaciones civiles de usuarios que se tuvieron que organizar y reestructurar en su interior para llevar a cabo el manejo y distribución del agua de riego, forma en que operan actualmente (Dávila et al., 1996). En la zona de estudio este proceso se llevó a cabo en 1992, con lo que se constituyeron legalmente dos asociaciones de usuarios.

1) Asociación de usuarios del distrito de riego $\mathrm{N}^{\circ} 044$. Se localiza en el municipio de Jilotepec, cuenta con la presa Danx- 
ho para la gestión del agua por parte de los productores y la irrigación de parcelas. Danxho inició su operación en 1951 y tiene una capacidad de 31'500,000 m³. De 1991 a 2003 se irrigaron 3,668.3 ha en promedio, se designó 74.4\% del riego al municipio de Jilotepec y $25.6 \%$ a Soyaniquilpan de Juárez. De acuerdo con información proporcionada por la CNA, de 1991 a 2003 se destinó 87.6\% del riego al cultivo de maíz, $1.9 \%$ al trigo, $1.5 \%$ en frutales, $4.5 \%$ a praderas y $4.5 \%$ a avena, lo que en conjunto representó 9\% del riego asignado a cultivos forrajeros. Al analizar la información a detalle, se encontró que en 1991 se reportaron 5,068 ha irrigadas de maíz y 3,229.1 ha para 2003, lo que implicó un decremento de $63.7 \%$ en un lapso de 12 años. En relación con los forrajes se presentó el caso contrario, un incremento de $43.9 \%$ para el mismo periodo.

2) Asociación de usuarios del distrito de riego $\mathrm{N}^{\circ} 096$ Arroyo Zarco. Se encuentra en el municipio de Aculco, cuenta con el Módulo Huapango y el Módulo II de Ñado, con las presas del mismo nombre para la captación y distribución del agua de riego. La presa Huapango tiene una capacidad de 121'000,000 de $\mathrm{m}^{3}$ e irriga los municipios de Aculco, Polotitlán y Jilotepec, además de una mínima parte de los municipios de Huichapan y Nopala en el estado de Hidalgo. El 90.7\% del riego se destinó al Estado de México y 9.3\% al de Hidalgo. Al municipio de Aculco se asignó 49.5\% del agua de riego, $35.3 \%$ en Polotitlán y 5.9\% en Jilotepec. La presa Nado tiene una capacidad de $16,500,000 \mathrm{~m}^{3}$ y la totalidad del riego se asigna al municipio de Aculco. Con las presas de este distrito de riego se irrigaron 6,751.5 ha en promedio de 1991 a 2003 . El riego se destinó en $83.7 \%$ al cultivo de maíz, $0.5 \%$ al trigo, $0.5 \%$ en frutales, $11.7 \%$ a praderas y $3.6 \%$ a avena, es decir, para cultivos forrajeros se asignó $15.3 \%$ del riego. Contrario al caso anterior, en este distrito se tuvo un incremento de la superficie irrigada de maíz, en 1991 se reportaron 6,279 ha y para 2003, $8,195.7$, lo que representó un incremento de $76.6 \%$. El riego para forrajes también aumentó, se reportó una superficie irrigada de 404.5 ha en 1991 y $1,067.8$ ha en 2003 , con un aumento de $37.9 \%$, disminuyendo el riego hacia otros cultivos como trigo y frutales. 


\subsubsection{Organización en el manejo del agua y características sociales de los usuarios del riego}

El manejo y medición del agua de riego que se asigna a las parcelas de los productores es responsabilidad en primera instancia de la CNA, la cual está a cargo del vaso de agua, determina el volumen a extraer y su distribución. Para el mantenimiento de las presas la CNA recibe $17 \%$ de lo recaudado por las asociaciones civiles de usuarios.

Una vez que el volumen de agua se determina y se pone en los canales principales de distribución, la responsabilidad recae en las asociaciones civiles de usuarios que distribuyen el agua a las diferentes comunidades, previo pago del servicio por parte de los representantes o canaleros.

La función de los representantes de cada comunidad es realizar el trámite del servicio de riego. Acuden a las oficinas de cada distrito para solicitar y pagar el agua, se extiende el recibo correspondiente y se otorga a cada productor de acuerdo con la cantidad solicitada.

El canalero es el distribuidor de agua que en ocasiones puede tramitar el servicio de riego o no, pero por lo general su función es canalizar el agua a las parcelas de los productores que hayan cubierto su cuota. La función colectiva de los usuarios respecto del manejo del agua es el desasolve de los canales de riego cada año, durante los meses de febrero a marzo.

La información generada en campo con las entrevistas a los usuarios mostró que el promedio de edad fue de 51 años. En relación con el nivel de escolaridad, la mayor parte de los usuarios tiene estudios de primaria (74.4\%), con secundaria y bachillerato los productores más jóvenes (13.4\%) y sólo $12.2 \%$ no tiene estudios.

El acceso al recurso tierra se obtuvo en $63.2 \%$ por sucesión de padres a hijos al fraccionar los terrenos, además de la herencia de otros familiares como los abuelos y tíos. La compra de terrenos representó 21.4\% y el reparto ejidal significó 15.4\%, manifestado por aquellos productores de mayor edad. Cabe mencionar que el reparto agrario comenzó en la zona de estudio a partir de 1921.

En relación con la tenencia de la tierra, la mitad de la superficie agrícola se encontró en régimen ejidal, la propiedad privada representó 21.4\%, 25.2\% lo manejan de manera mixta (ejidal y privada) y la propiedad comunal significó 3.4\%, no obstante, cada 
productor trabaja sus parcelas de manera individual y dicen que no cuentan con un título que ampare su propiedad, por lo que la declaran como comunal.

La principal forma que tienen los productores de acceder al riego constituyó la sucesión de padres a hijos y representó 84.7\%, el trámite directo del servicio significó 6.9\%, el acceso mediante reparto ejidal $2.7 \%$ y $5.7 \%$ por aquellos productores que acceden al agua de riego mediante la compra y renta de terrenos que están registrados ante la CNA con derechos, así como productores eventuales que reciben agua de acuerdo con la disponibilidad en las presas.

Del total de productores que hacen uso del agua de riego, 91.5\% obtuvo el agua a partir de las presas que pertenecen a los distritos de riego de la zona (Huapango, Nado y Danxho). Un $3.1 \%$ la obtuvo de la presa la Goleta, ubicada en el municipio de Soyaniquilpan de Juárez y el 5.4\% restante se derivó de otras presas distribuidas al interior de la región catalogadas como unidades de riego.

\subsubsection{Usos del agua de riego y actividad agropecuaria}

El uso del agua de riego sigue siendo principalmente para cultivos básicos. El 47\% de las unidades de producción la destinaron al cultivo de maíz, con un riego al año y otro como auxilio en planta sólo en caso de disponer de agua en las presas, conocido por los productores como punta de riego. El 19.8\% lo dedicó a praderas con un promedio de cinco riegos por año, 7.2\% a la avena con dos riegos en promedio por año, $2.7 \%$ se distribuyó entre el cultivo del frijol, haba, trigo, cebada o frutales, también como punta de riego, y una parte considerable de los productores (23.4\%) manifestó que lo utilizó para abrevadero. Este último no lo usan algunos productores ya que cuentan con agua potable en sus domicilios, tienen a sus animales estabulados y además se requiere contar con bordos para la captación del agua.

De forma general los usos del riego se enfocaron a cuatro rubros: maíz, praderas que en su mayoría son una combinación de pasto, trébol o alfalfa, avena y agua para abrevar al ganado en los meses más secos del año.

En relación con la actividad agrícola, los productores contaron en promedio con 4.7 ha de las cuales 3.3 fueron irrigadas, ya que el resto correspondió a la superficie de temporal y otras tierras que se aprovechan como potrero por la dificultad para sem- 
brar cultivos. El promedio de tierra por productor se incrementó ya que al inicio de la actividad la superficie utilizada era de 3.4 ha, sin embargo, a través de la compra y renta de terrenos con derecho a riego, así como la necesidad de contar con forrajes para alimentar a los animales, dicha superficie ha aumentado.

En cuanto a la superficie destinada a la siembra de cultivos (1'121.4 ha), 74.6\% se designó al maíz que sembraron 95.8\% de los productores, pero debido al bajo precio que presenta en el mercado se ha preferido dar a los animales, aprovechando además los subproductos como el rastrojo y el grano. Comparando el destino del maíz que constituyó el eje agropecuario de la región, se obtuvo que la venta del maíz disminuyó de 28.2\% al inicio de la actividad agropecuaria a sólo $11.8 \%$ actualmente. En opinión de los productores, antes aprovechaban la presencia de Conasupo (Consejo Nacional de Subsistencias Populares) para colocar los excedentes y ahora sólo se vende una pequeña parte de la producción para recuperar la inversión en los trabajos de cultivo y contar con dinero para el siguiente ciclo. El autoconsumo también mostró un decremento, al inicio captaba 52.2\% de lo producido y actualmente $40.5 \%$.

La tendencia hacia el uso pecuario se incrementó, sobre todo en la alimentación de vacas lecheras, ya que al inicio de la actividad se destinaba $16.5 \%$ del maíz producido a los animales y actualmente 41.1\%. El uso en la alimentación de otros animales (cerdos, borregos, caballos y asnos) también mostró un incremento de $3 \%$ a $6.5 \%$. De las 836.15 ha de maíz que se cultivaron en total, $47.7 \%$ se destinó a la actividad pecuaria y el resto se distribuyó entre la venta del grano y autoconsumo.

Lo anterior en función de que 92.4\% de los productores contaron con la integración agrícola-pecuaria, sólo $6.5 \%$ destinó sus tierras a la actividad netamente agrícola y a la actividad ganadera $1.1 \%$.

Sólo $1.5 \%$ de los productores sembró trigo, lo que representó 1-6\% de la superficie total. El frijol lo cultivaron 4.2\% de los productores y ocupó $0.9 \%$ de la superficie sembrada. En cuanto a la cebada, 2.3\% de los productores que utilizaron riego la produjeron y se destinó $3.7 \%$ de la superficie total cultivada.

En forrajes se designó $18.8 \%$ de la superficie total cultivada, considerando praderas, alfalfa y avena. De las 211.5 ha que se emplearon para producir estos forrajes, $87.4 \%$ se dedicó a la alimentación de vacas lecheras, 9.2\% a otros animales y sólo 3.4\% a la venta a otras personas. 


\section{Cuadro 1}

Destino de la producción de maíz

\begin{tabular}{lcc}
\hline \multicolumn{1}{c}{ Destino } & $\begin{array}{c}\text { Inicio de la actividad } \\
\text { porcentaje }\end{array}$ & $\begin{array}{c}\text { Actual } \\
\text { porcentaje }\end{array}$ \\
\hline Venta & 28.2 & 11.8 \\
Autoconsumo & 52.2 & 40.5 \\
Vacas & 16.5 & 41.1 \\
Otros animales & 3.0 & 6.5 \\
\hline
\end{tabular}

Fuente: Elaboración propia con información de campo.

Si se extrapola esta información a superficie agrícola, 47.7\% de la superficie del maíz se orientó a la producción animal, lo que representó $35.5 \%$ de la superficie total cultivada, sumado al $18.8 \%$ que se destinó a la superficie sembrada con forrajes, equivale a $54.3 \%$ de la superficie total cultivada destinada a la producción animal.

Aunado a lo anterior, de 1991 a 2003 la CNA reportó que el agua de riego se destinó en $86.7 \%$ al cultivo de maíz, 9.3\% a praderas y $4 \%$ a la avena. En campo se encontró que el porcentaje para maíz disminuyó a 78.7\% y se incrementó en forrajes con $12.8 \%$ a praderas y $8.4 \%$ a avena.

Cabe mencionar que la renta de tierras no fue relevante, $18.3 \%$ de los productores que hacen uso del riego rentan tierra a otras personas con la finalidad de abastecerse de forraje para los animales y obtener maíz para el consumo familiar, mientras que sólo $1.1 \%$ de los usuarios del riego rentan parte de sus tierra a otras personas y representó $0.6 \%$ de la superficie total. De los 48 productores que participan en la transferencia de tierra, de un total de 262 muestreados, $22.9 \%$ trabajó la tierra a medias, es decir, un productor proporciona el terreno agrícola y los insumos, mientras que el otro aporta su trabajo, al final se reparten lo producido.

La CNA estableció el costo de los diferentes cultivos de acuerdo con el distrito de riego y al realizar la comparación con los resultados en campo, mostraron que lo que pagan los productores es ligeramente mayor. Para el caso del maíz, el costo oficial fue de $\$ 55.00$ en promedio y en campo los productores reportaron una erogación en efectivo de $\$ 75.00$. La irrigación de praderas tuvo un costo promedio de $\$ 73.40$ y en campo de $\$ 98.80$. Para el cultivo de avena la CNA consideró $\$ 73.40$ y el productor pagó \$ 86.80, no obstante, los representantes y canaleros reciben una cuota extra por el trámite del servicio. Para el abrevade- 
ro el costo reportado en campo es menor en comparación con lo que manifestó la CNA, sin embargo, los productores obtienen el recurso agua a partir de lo pagado por el riego de cultivos básicos o forrajeros y lo almacenan en bordos para su uso posterior, de esta forma se ahorran el gasto del agua para los animales.

Se calcularon los ingresos por concepto de riego de cultivos (se dejó fuera el abrevadero por la dificultad para estimarse). Considerando el número de riegos por cultivo, así como una muestra de 5\% en campo, se encontró que la erogación en efectivo por parte de los productores es mayor a lo que informa la $\mathrm{CNA}$, lo que demuestra la cantidad de ingresos que pierde la institución y las asociaciones de usuarios, ya que el trámite del servicio de riego no se hace de manera directa por productor, sino a través de intermediarios. En relación con el costo por cultivo, para el caso del maíz y la avena se irrigan con punta de riego, en comparación, las praderas se irrigan cinco veces en promedio al año, por lo que el desembolso para los cultivos forrajeros es mayor que en cultivos básicos. A partir de los cálculos que se realizaron, tomando en cuenta el tipo de cultivo, número de riegos y el costo por distrito, se encontró que la CNA recaudó \$ 989,390.50 durante el año y lo erogado por los productores ascendió a \$2’003,658.00, por lo que la diferencia entre lo que captó la CNA y lo realmente pagado por el productor se reparte entre los distintos representantes que tramitan el servicio.

Respecto de la actividad pecuaria, destacó la producción de leche que representó una de las actividades más importantes para los productores que hacen uso del agua de riego en la región noroeste. El 59.2\% de los usuarios se dedicaron a esta actividad debido a que cuentan con agua para el establecimiento de cultivos forrajeros, necesarios en la alimentación de vacas lecheras. Si bien $40.8 \%$ de los productores no trabajan la producción de leche de manera formal, $18.3 \%$ contó con una o dos vacas, sin embargo, se consideró que no pueden depender directamente de la actividad lechera con este número de animales, pues el producto fue para autoconsumo de la familia y la alimentación de las crías de las vacas. El número de animales mostró un incremento considerable, cuando estos productores se iniciaron en la actividad agropecuaria contaban con un promedio de dos vacas y actualmente con cinco.

La producción de leche representó un ingreso y negocio adecuados, así lo manifestaron 33.7\% de los productores, 31.4\% declararon que no contaban con otra opción de trabajo en la 
Cuadro 2

Cálculo de los ingresos generados por concepto de riego

\begin{tabular}{|c|c|c|c|c|c|c|c|}
\hline & Cultivo & & $\begin{array}{c}\text { Costo por } \\
\text { riego } \\
\text { (pesos) }\end{array}$ & $\begin{array}{c}N^{\circ} \text { de } \\
\text { hectáreas } \\
\text { irrigadas }\end{array}$ & $\begin{array}{c}\text { Riegol } \\
\text { hectárea }\end{array}$ & Total & $\begin{array}{c}\text { Pesos } \\
\text { por } \\
\text { cultivo }\end{array}$ \\
\hline \multirow[t]{3}{*}{$\mathrm{CNA}$} & Maíz & $*$ & $\begin{array}{l}65.0 \\
45.4\end{array}$ & $\begin{array}{l}3,229.1 \\
8\end{array}$ & 1 & $\begin{array}{l}209,893.4 \\
371759 ?\end{array}$ & $581,652.6$ \\
\hline & Pradera & $*$ & 82.0 & 150.0 & 5 & 61,500.0 & $372,540.0$ \\
\hline & Avena & $\begin{array}{l}* * \\
* \\
* *\end{array}$ & $\begin{array}{l}64.8 \\
82.0 \\
64.8\end{array}$ & $\begin{array}{l}960.0 \\
344.0 \\
107.7\end{array}$ & 1 & $\begin{array}{r}311,040.0 \\
28,213.7 \\
6,984.1\end{array}$ & $35,197.8$ \\
\hline \multicolumn{2}{|c|}{ Total CNA } & & & & & & $989,390.5$ \\
\hline \multirow[t]{3}{*}{ Campo } & Maíz & $\begin{array}{l}* \\
* * \\
* * *\end{array}$ & $\begin{array}{l}65.8 \\
81.5 \\
78.3\end{array}$ & $\begin{array}{r}178.5 \\
323.2 \\
96.7\end{array}$ & 1 & $\begin{array}{r}11,745.3 \\
26,344.8 \\
7,575.5\end{array}$ & $45,665.6$ \\
\hline & Pradera & $\begin{array}{l}* \\
* * \\
* * *\end{array}$ & $\begin{array}{r}86.5 \\
105.6 \\
104.4\end{array}$ & $\begin{array}{l}19.0 \\
49.2 \\
28.7\end{array}$ & 5 & $\begin{array}{r}8,217.5 \\
26,004.0 \\
15,007.5\end{array}$ & $49,229.0$ \\
\hline & Avena & 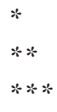 & $\begin{array}{l}73.2 \\
98.2 \\
89.0\end{array}$ & $\begin{array}{r}39.2 \\
18.2 \\
7.0\end{array}$ & 1 & $\begin{array}{r}2,873.1 \\
1,792.1 \\
623.0\end{array}$ & $5,288.2$ \\
\hline \multicolumn{2}{|c|}{ Total Campo } & & & & & & $100,182.9$ \\
\hline
\end{tabular}

Fuente: Elaboración propia con información de campo y CNA, 2003.

*Danxho, ** Huapango, *** Nado. En el caso del distrito de riego 096, la CNA reportó el número de hectáreas de ambos módulos de riego, Huapango y Ñado, por lo que no fue posible la separación como en el trabajo de campo.

zona, ya que trabajos remunerados mediante un salario en la industria local no les alcanzaría para mantener a sus familias, un grupo más reducido dijo que la actividad es tradicional y otros que es un medio de integración a la agricultura, por lo que aprovechan mejor lo producido.

\section{Conclusiones}

Las zonas de bosques han favorecido la presencia de lluvias, por lo que la creación de infraestructura en función de las presas y canales de distribución de agua de riego han sido fundamentales para los cultivos básicos y forrajeros, incluso en áreas de menor precipitación pluvial como el municipio de Polotitlán, norte de Aculco y Soyaniquilpan de Juárez, beneficiando zonas que de manera natural presentan limitantes para la irrigación de cultivos, contribuyendo al desarrollo de la producción agropecuaria.

La zona mostró su tendencia a la actividad agrícola y pecuaria desde la presencia de las distintas haciendas dedicadas a la siem- 
bra de maíz o trigo como cultivos básicos y el pastoreo de animales en pastizales naturales, no obstante, si bien se ha modificado la forma de producción, el patrón de cultivos se mantiene hasta la actualidad.

En la región predominaron explotaciones en pequeña escala que utilizaron el agua de riego por gravedad para la producción de cultivos básicos y forrajeros. De los primeros, el maíz constituyó el eje agropecuario de la región y de acuerdo con la estrategia de las unidades de producción se ha preferido integrarlo a la actividad pecuaria. Lo anterior en función de los resultados de este trabajo que muestran que la venta y autoconsumo del grano disminuyó, mientras que el destino pecuario se incrementó. David Barkin (2003) mencionó que el maíz irrigado presenta una productividad 2.5 veces mayor que el de temporal y ha sido el cultivo más intensivo en el uso del agua en el país, situación similar se encontró en la región de estudio.

El establecimiento de forrajes ha sido posible porque se cuenta con varias presas al interior de la región, las cuales captan el agua durante la estación de lluvias y permiten su uso en la temporada seca, lo que facilita el aprovechamiento del terreno agrícola y la integración con la actividad pecuaria. Lo anterior debido al establecimiento de cultivos como la avena que es de ciclo corto, sembrado en los terrenos cosechados de maíz, praderas que no requieren mayor inversión que la inicial y la utilización de subproductos de los cultivos básicos como el grano y rastrojo de maíz o pajas de frijol, trigo y cebada.

El número de bovinos manifestó su mayor incremento a partir de la década de los sesenta, lo cual se dio a partir de diversos factores: las diferentes acciones gubernamentales, federales y estatales, así como las locales a través de la organización de productores que han repercutido en la construcción de infraestructura de riego, establecimiento, producción de forrajes y una actividad lechera importante, situación que se reflejó en las cifras de los censos agrícolas y pecuarios, además de lo manifestado por los productores en las unidades de producción. En este sentido, el agua de riego se ha convertido en un factor estratégico para la producción de leche, pues ha posibilitado que se cuente con cultivos forrajeros como alimentos principales de los hatos bovinos que constituyeron el eje pecuario de la zona de estudio.

La CNA planteó el uso de riego para la actividad eminentemente agrícola, los resultados de este trabajo mostraron que se redujo debido a la inclusión de forrajes, sin embargo, si se considera 
el maíz destinado a la producción animal, el decremento es aún mayor, por lo que se puede concluir que en la zona se ha dado un proceso de integración agrícola-pecuaria del riego.

La organización en el manejo del agua es esencial para el riego de los cultivos, ya que permite instrumentar reglas que regulan el acceso y uso del agua, además de las tareas de mantenimiento de los canales.

La superficie que se irriga cada año puede variar y se debe considerar el incremento de agua para cultivos forrajeros que demandan mayor cantidad, el desasolve de las presas que limita la cantidad de agua captada por las mismas y que el número de riegos depende de los niveles de almacenamiento, aunado a la

problemática en el ordenamiento de usos y derechos, ya que el número de usuarios puede incrementarse, pero no así la superficie de riego, lo que limita la incorporación de nuevos productores para acceder a riego en sus parcelas e incrementar los rendimientos de sus cultivos.

De acuerdo con los costos del riego que estableció la CNA y lo que erogaron en campo los productores, se encontró que hay gran cantidad de dinero que se distribuye entre los distintos intermediarios que realizan la gestión del servicio, por lo que una opción que podría plantearse es que el trámite se realice a través de la dependencia por medio de técnicos y que éstos a su vez capaciten a los productores en cuanto a los requerimientos de agua por cultivo, lámina de riego a utilizar y el aprovechamiento de los distintos cultivos en los animales, así como generar una base de datos y estadísticas más acordes con la realidad que pudieran llevar a una gestión más eficiente en el uso del agua de riego.

\section{Bibliografía}

Aguado-López, Eduardo (1998), "El reparto ejidal en la década de los años veinte" en Una Mirada al Reparto Agrario en el Estado de México (1915-1992), De la Dotación y Restitución a la Privatización de la Propiedad Social, El Colegio Mexiquense, Zinacantepec, Estado de México, México, pp. 68-81.

Anaya-Brondo, Abelardo (1975), "Desarrollo de las áreas de riego en México durante los últimos cincuenta años", Secretaría de Recursos Hidráulicos, documento interno, México. 
Arredondo-Muñozledo, Benjamín (1971), Historia de la Revolución mexicana, Porrúa, México.

Barkin-Rappaport, David (2003), "La soberanía alimentaría: el quehacer del campesinado mexicano", Estudios Agrarios, Revista de la Procuraduría Agraria, año 9, 22, pp. 35-65.

CNA (Comisión Nacional del Agua) (2003), "Distrito de Riego 044 'Jilotepec, México’ y 096 'Arroyo Zarco’”, Jefatura de Distritos de Riego, Subgerencia de Ingeniería, Gerencia en el Estado de México, documento interno.

Cordero-Salas, Paula, Hugo Chavarría, Rafael Echeverri y Sergio Sepúlveda (2003), "Territorios rurales, competitividad y desarrollo", Cuaderno Técnico, núm. 23, IICA, Costa Rica, pp. 1-17.

Daniel-Wayne, W. (2004), "Muestras aleatorias y no aleatorias", en Bioestadística, base para el análisis de las ciencias de la salud, Limusa Wiley, México.

Dávila, Sonia, Ana Helena Treviño y Sergio Vargas (1996), “Gestión integral del recurso hidráulico a nivel cuenca: el caso de la cuenca del río la Laja", en Salvador Rodríguez, Margarita Camarena y Jorge Serrano (coords.), El desarrollo regional en México, antecedentes y perspectivas, Universidad Nacional Autónoma de México, México, pp. 765-783.

DGE (Dirección General de Estadística) (1937), Primer censo agrícola ganadero, 1930, DGE-Secretaría de la Economía Nacional, México.

DGE (Dirección General de Estadística) (1956), Tercer censo agrícola ganadero y ejidal, 1950, DGE-Secretaría de Economía, México.

DGE (Dirección General de Estadística) (1965), IV censo agrícola ganadero y ejidal, 1960, DGE-Secretaría de Industria y Comercio, México.

DGE (Dirección General de Estadística) (1970), v censo agrícola ganadero y ejidal, DGE, México. 
Espinoza-Ortega, Angélica (2004), Reestructuración de la lechería en la región noroeste del Estado de México en el marco del proceso de globalización, Universidad Nacional Autónoma de México, México.

FAO (Organización de las Naciones Unidas para la Agricultura y la Alimentación) (2004), "Superficie agrícola de riego", http://www.faostat.fao.org/faostat/servlet/, 29 de septiembre de 2004.

Gómez-Zepeda, Ignacio (1994), Historia de las unidades de riego. Memorias de un soñador, Centro de investigaciones y Estudios Superiores en Antropología Social-Comisión Nacional del Agua, México.

Gómez-Oliver, Luis (1996), "El papel de la agricultura en el desarrollo de México", Estudios Agrarios, Revista de la Procuraduría Agraria, año 2, 3, México, pp. 33-84.

INEGI (Instituto Nacional de Estadística, Geografía e Informática) (1994), VII censo agrícola y ganadero, resultados definitivos, t. I-III, INEGI, México.

INEGI (Instituto Nacional de Estadística, Geografía e Informática) (1997), El maíz en el Estado de México, INEGI, México.

INEGI (Instituto Nacional de Estadística, Geografía e Informática) (2000), Carta temática. Escala 1:50,000, sistemas digitales, INEGI, México.

Morales-Zafra, Carlos (1998), Soyaniquilpan de Juárez, monografía municipal, Gobierno del Estado de México-Instituto Mexiquense de Cultura-Asociación Mexiquense de Cronistas Municipales, México.

Salinas-Sandoval, María del Carmen (2001), "Problemas por tierras de los pueblos de la municipalidad de Jilotepec, siglo XIX", Documentos de Investigación, El Colegio Mexiquense, México, pp. 1-17.

Sedagro (Secretaría de Desarrollo Agropecuario) (2000a), "Series estadísticas por municipio en los últimos diez años", 
documento interno, Delegación Estado de México, Distrito de Desarrollo Rural, Jilotepec, México.

Sedagro (Secretaría de Desarrollo Agropecuario) (2000b), "Uso del suelo en el Estado de México”, documento interno, Delegación Regional Nº viII, Jilotepec, México.

Segura-Correa, José y Nicholas Honhold (1993), Manual de muestreo para la salud y producción animal, Universidad Autónoma de Yucatán, Mérida.

Vargas, Sergio (2002), “Agua y agricultura: paradojas de la gestión descentralizada de la gran irrigación”, Estudios Agrarios, Secretaría de la Reforma Agraria, año 8, 20, México, pp. 61-81.

Villagómez-Velásquez, Yanga (2002), "Gestión social del agua y cambio agrario en el Istmo de Tehuantepec, Oaxaca", Estudios Agrarios, Revista de la Procuraduría Agraria, año 8, 20, México, pp. 83-123.

Villalobos-López, Gonzalo (2000), “Desarrollo local y reforma agraria en México. Retos y perspectivas en la globalización económica", Estudios Agrarios, Revista de la Procuraduría Agraria, año 6, 15, México, pp. 133-171.

Zetina, Francisco Javier (2002), "Foros de estudios agrarios", Revista de la Procuraduría Agraria, año 8, 20, México, pp. 141-143.

Recibido: 8 de mayo de 2006. Reenviado: 8 de mayo de 2007. Aceptado: 20 de septiembre de 2007.

Tirzo Castañeda Martínez. Es maestro en el programa de Ciencias Agropecuarias y Recursos Naturales de la Universidad Autónoma del Estado de México (UAEM). Sus estudios de licenciatura los realizó en la Facultad de Medicina Veterinaria y Zootecnia de la UAEM. Actualmente cursa el último semestre de doctorado en el programa de Ciencias Agropecuarias y Recursos Naturales de la UAEM. Su línea de investigación es producción animal cam- 
pesina. Es coautor del artículo "Small-Scale Dairy Farming in the Highlands of Central Mexico: Technical, Economic and Social Aspect and Their Impact on Poverty", Expt Agric, 43, Cambridge University Press, pp. 1-16 (2007).

Sergio Franco Maass. Es doctor en Geografía por la Universidad de Alcalá (España); es investigador del Centro de Investigación en Ciencias Agropecuarias de la UAEM. Su línea de investigación es conservación y manejo de recursos naturales. Entre sus publicaciones más recientes se encuentran: "Territorialidad y política. El caso de la división del municipio de San Felipe del Progreso, Estado de México", Gestión y Política Pública, xvi(2), pp. 163-206 (2007); en coautoría, "Payments for Environmental Services: An alternative for Sustainable Rural Development?", Mountain Research and Development, 28(1), pp. 23-25 (2008); en coautoría, "Estimación del valor económico del servicio ambiental de captura de carbono en los bosques templados del Estado de México (México)", en Juan Gallardo (comp.), La captura de carbono en ecosistemas terrestres Iberoamericanos, Sociedad Iberoamericana de Física y Química Ambiental, Salamanca, España, 2008.

Carlos González Esquivel. Es doctor en filosofía y agroecología por la Universidad de Londres. Actualmente es investigador del Centro de Investigación en Ciencias Agropecuarias de la UAEM, Sus líneas de investigación son agroecología y evaluación de sustentabilidad en agroecosistemas. Entre sus últimas publicaciones se encuentran: "Agroecología y sustentabilidad", Convergencia, 46, Universidad Autónoma del Estado de México, México, pp. 51-87 (2008); en coautoría, "A methodology for evaluating the sustainability of inland wetland systems", publicado en línea en Aquaculture International, DOI 10.1007/s10499-007-9163-5, en coautoría, "Payments for Environmental Services: An alternative for Sustainable Rural Development?", Mountain Research and Development, 28 (1), pp. 23-25 (2008).

Angélica Espinoza Ortega. Es doctora en ciencias por la Universidad Nacional Autónoma de México (UNAM); maestra en desarrollo rural regional por la Universidad Autónoma Chapingo y médico veterinario zootecnista por la Universidad Autónoma del Estado de México (UAem). Pertenece al Sistema Nacional de Investigadores (SNI), nivel I. Se desempeña como investigadora del Centro de Investigación en Ciencias Agropecuarias de la UAEM. Sus líneas 
de investigación son: desarrollo rural regional, socioeconomía campesina, sistemas de producción de leche y problemática lechera. Entre sus publicaciones recientes se encuentran: "Small-scale dairy farming in the highlands of central Mexico: Technical, economic and social aspects and their impact in poverty", Experimental Agriculture, 47, pp. 1-16, 2007; "Annual variation in economics of small-scale dairy production and its effect on poverty indexes", Journal of Livestock Research and Rural Development, 2(20) 2008; Los quesos mexicanos genuinos, un patrimonio cultural que se debe rescatar, Mundi Prensa, UACH-UAEM, México (2008); Agroindustria rural y territorio, $t$. II, ed. en C3, UAEM-IICA-CIRAD, Universidad de Versalles-syal, México, (2007). 